\title{
Creation and annihilation of free volume during homogeneous flow of a metallic glass
}

\section{Citation}

Heggen, Marc, Frans Spaepen, and Michael Feuerbacher. 2005. "Creation and Annihilation of Free Volume during Homogeneous Flow of a Metallic Glass." Journal of Applied Physics97 (3): 033506. https://doi.org/10.1063/1.1827344.

\section{Permanent link}

http://nrs.harvard.edu/urn-3:HUL.InstRepos:41511254

\section{Terms of Use}

This article was downloaded from Harvard University's DASH repository, and is made available under the terms and conditions applicable to Other Posted Material, as set forth at http:// nrs.harvard.edu/urn-3:HUL.InstRepos:dash.current.terms-of-use\#LAA

\section{Share Your Story}

The Harvard community has made this article openly available.

Please share how this access benefits you. Submit a story.

Accessibility 


\title{
Creation and annihilation of free volume during homogeneous flow of a metallic glass
}

\author{
Marc Heggen \\ Institut für Festkörperforschung, Forschungszentrum Jülich, D-52425 Jülich, Germany \\ Frans Spaepen ${ }^{\mathrm{a})}$ \\ Division of Engineering and Applied Sciences, Harvard University, Cambridge, Massachusetts 02138 \\ Michael Feuerbacher \\ Institut für Festkörperforschung, Forschungszentrum Jülich, D-52425 Jülich, Germany
}

(Received 13 July 2004; accepted 5 October 2004; published online 30 December 2004)

\begin{abstract}
Bulk samples of $\mathrm{Pd}_{41} \mathrm{Ni}_{10} \mathrm{Cu}_{29} \mathrm{P}_{20}$ glass were tested at constant true stress (20-636 MPa) in compression at 550,555 , and $565 \mathrm{~K}$ to study the transition from steady-state creep at low stress to deformation-induced softening at high stress. In the high-stress regime strongly accelerating creep was observed. All deformation was homogeneous. The activation volume was $106 \AA^{3}$. The isoconfigurational activation energy was $9.03 \pm 0.64 \mathrm{eV}$. The disordering rate is proportional to the applied power density (stress $\times$ strain rate). The disordering process is inefficient in energy and in the production of free volume. (C) 2005 American Institute of Physics. [DOI: 10.1063/1.1827344]
\end{abstract}

\section{INTRODUCTION}

Plastic deformation of metallic glasses has two modes. ${ }^{1,2}$ At low temperature and high stress, these glasses deform inhomogeneously, i.e., the deformation is confined to a small number of very thin shear bands. Inside these bands, the shear strain and shear strain rate are very large. At higher temperature and lower stress, the deformation is homogeneous, i.e., each volume element of the material undergoes the same strain. The latter mode includes flow of the liquid.

Inhomogeneous flow is an instability due to progressive softening brought about by deformation-induced disordering. ${ }^{3}$ The atomistic mechanism of the disordering process is still under investigation. For example, it has been variously proposed that the disordering depends on the strain, the strain rate or the stress. ${ }^{4-12}$ The functional dependence of the disordering process on these quantities is a fortiori unknown. It seems clear, though, that the process is accompanied by shear-induced dilatation, ${ }^{13-16}$ a phenomenon that has been known for years in the deformation of soils that consist of randomly packed grains. ${ }^{17,18}$

One expects deformation-induced disordering to occur during homogeneous deformation as well. Most of the experiments in this mode, however, have been performed at low stresses and strain rates, where the disordering rate is small and is overwhelmed by the ordering process known as structural relaxation. ${ }^{2,19-23}$ In the experiments that have been carried out in the high-stress part of the homogeneous mode, a disordering process has indeed been identified. De Hey, Siestma, and van den Beukel made such observations using tensile tests at constant strain rate on $\mathrm{Pd}_{40} \mathrm{Ni}_{40} \mathrm{P}_{20}{ }^{6}$ The disordering was most apparent from the dramatic drop in the flow stress in many tests. Lu, Ravichandran, and Johnson made similar observations on the Zr-based Vitreloy-1 alloy. ${ }^{8}$

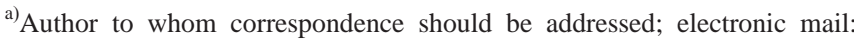
spaepen@deas.harvard.edu
}

Heggen, Spaepen, and Feuerbacher ${ }^{9,10}$ have used compression creep testing at constant stress to study this regime in $\mathrm{Pd}_{41} \mathrm{Ni}_{10} \mathrm{Cu}_{29} \mathrm{P}_{20}$, and observed accelerating strain rates. The advantage of studying disordering in the homogeneous flow mode is that the process is sufficiently slow for its functional dependence on stress, strain rate, or strain to be established. In this paper these dependences are analyzed on creep data on $\mathrm{Pd}_{41} \mathrm{Ni}_{10} \mathrm{Cu}_{29} \mathrm{P}_{20}$.

\section{THE FREE VOLUME MODEL}

Plastic deformation of metallic glasses occurs by the superposition of the shear of localized groups of atoms, often referred to as "flow defects" on "shear transformation zones." Subjected to an external stress $\sigma$, small regions of volume $\nu_{0}$ undergo a strain $\varepsilon_{0}$ at a rate that depends on the stress. If the concentration of these flow defects is $c_{f}$, the macroscopic strain rate can be written as

$$
\frac{d \varepsilon}{d t}=2 c_{f} k_{f} \frac{\varepsilon_{0} \nu_{0}}{\Omega} \sinh \left(\frac{\varepsilon_{0} \nu_{0} \sigma}{2 k T}\right)
$$

where $k_{f}$ is a temperature-dependent rate constant, $\Omega$ is the atomic volume, $k$ is Boltzmann's constant, and $T$ is the temperature. ${ }^{1}$ The product $\varepsilon_{0} \nu_{0}$ is the activation volume of the process. The stresses $\sigma$ and strains $\varepsilon$ in this analysis are taken to be uniaxial quantities, as in the compression tests to be performed. Since plastic deformation is a shear process, similar equations in terms of shear stresses and shear strains are often found in the literature.

The defects in amorphous metals are often described by the free volume model, ${ }^{24}$ in which they are density fluctuations with volume greater than a critical value $\nu^{*}$. The defect concentration is described by 


$$
c_{f} \equiv \exp \left(-\frac{\gamma \nu^{*}}{\nu_{f}}\right)=\exp \left(-\frac{1}{x}\right),
$$

where $\gamma$ is a geometrical overlap factor between 0.5 and 1 , and $\nu_{f}$ is the average free volume per atom. The quantity $x$ $=\nu_{f} / \gamma \nu^{*}$ is the reduced free volume.

The annihilation of defects during structural relaxation takes place by a bimolecular process, the rate of which can be described as

$$
\frac{d c_{f}}{d t} \mid=-k_{r} c_{f}^{2}
$$

where $k_{r}$ is a rate constant. The validity of this ansatz has been verified by measurements of the viscosity, which at low stresses is inversely proportional to $c_{f}$. During structural relaxation, the viscosity increases linearly with time as implied by Eq. (3). ${ }^{19,25}$ The bimolecular nature of the annihilation process is easily understood in the case of amorphous $\mathrm{Si}^{26}$ for example, where annihilation occurs by the joining of two dangling bonds. In metallic glasses, the physical basis of the bimolecular reaction is less obvious. One interpretation is that the juxtaposition of two fairly equiaxed voids (density fluctuations) creates one with a larger aspect ratio that can collapse under the interatomic tractions on its surface (such as a vacancy disk in crystals) and thereby displace the free volume elastically to the sample boundary. ${ }^{27}$

Tsao and Spaepen, Volkert and Spaepen, and Duine, Sietsma, and van den Beukel have shown that during isothermal annealing at temperatures close to the glass transition temperature $T_{g}$ the viscosity of amorphous metals reaches a saturation value. ${ }^{19-21,28}$ Since the viscosity is inversely proportional to the defect concentration $c_{f}$, the defect concentration then reaches a metastable equilibrium concentration $c_{\mathrm{eq}}$. Duine, Sietsma, and van den Beukel found from the fitting of viscosity and calorimetric data that the change of $c_{f}$ towards equilibrium can best be described by an appropriately adapted bimolecular rate equation ${ }^{28}$

$$
\left.\frac{d c_{f}}{d t}\right|^{-}=-k_{r} c_{f}\left(c_{f}-c_{\mathrm{eq}}\right) \text {. }
$$

The deformation-driven creation of defects can be modeled through the free volume theory by considering the density fluctuations with volumes just smaller than the critical size $\nu^{*}$. Under stress, a neighboring atom can be squeezed into this pocket, the expansion of which leads to the creation of free volume. The resulting expression for the creation of free volume is ${ }^{4}$

$$
\frac{d \nu_{f}}{d t}=2 c_{f} k_{f} \frac{k T}{S} \frac{\gamma \nu^{*}}{\nu_{f}}\left[\cosh \left(\frac{\sigma \varepsilon_{0} \nu_{0}}{2 k T}\right)-1\right],
$$

where $S$ is a stiffness, obtained from Eshelby's theory for the dilatation of an isotropic continuum ${ }^{29}$

$$
S=\frac{2}{3} \mu \frac{1+\nu}{1-\nu},
$$

where $\mu$ and $\nu$ are, respectively, the shear modulus and Poisson ratio. At high stress, the hyperbolic functions in Eqs. (1) and (5) approximate exponentials, and Eq. (5) reduces to

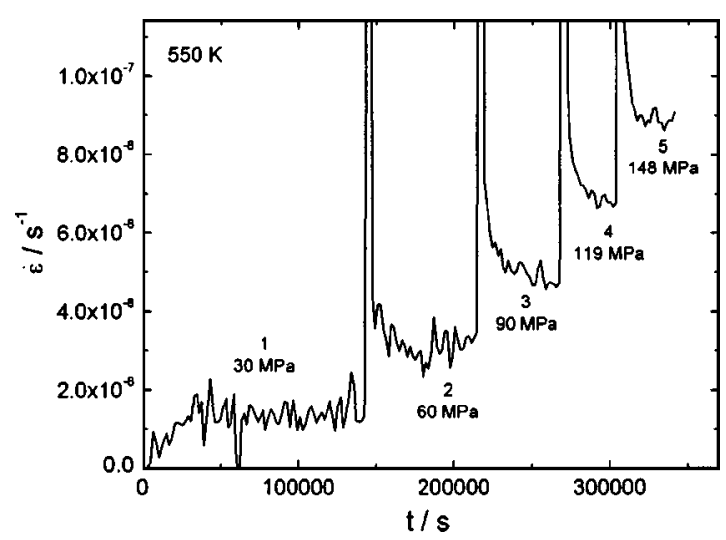

FIG. 1. Strain rates measured in a uniaxial compression creep test on a $\mathrm{Pd}_{41} \mathrm{Ni}_{10} \mathrm{Cu}_{29} \mathrm{P}_{20}$ metallic glass at $550 \mathrm{~K}$ at constant true stresses of 30,60 , 90,119 , and $148 \mathrm{MPa}$. The numbers indicate the order of the test.

$$
\frac{d \nu_{f}}{d t}=\frac{k T}{S} \frac{\gamma \nu^{*}}{\nu_{f}} \frac{\varepsilon_{0} \nu_{0}}{\Omega} \frac{d \varepsilon}{d t} \equiv a_{x} \gamma \nu * \frac{d \varepsilon}{d t} .
$$

This proportionality between defect creation rate and the strain rate has been used in the data analysis of de Hey, Sietsma, and van den Beukel. ${ }^{6}$ At low stress, expansion of the sinh in Eq. (1) and the cosh in Eq. (5) leaves a term that is, respectively, linear and quadratic in the stress. Combination of the two results leads to the following expression for Eq. (5):

$$
\frac{d \nu_{f}}{d t}=\frac{1}{8} \frac{\Omega}{S} \frac{\gamma \nu^{*}}{\nu_{f}} \frac{d \varepsilon}{d t} \sigma \equiv a_{x}^{\prime} \gamma \nu * \frac{d \varepsilon}{d t} \sigma .
$$

Here, the defect rate is proportional to the product of stress and strain rate, i.e., the applied power density. ${ }^{5}$ In the analysis of the data from the experiments described below, these two limits will be considered.

\section{EXPERIMENTS}

Metallic glass samples of composition $\mathrm{Pd}_{41} \mathrm{Ni}_{10} \mathrm{Cu}_{29} \mathrm{P}_{20}$ and size $5 \times 2 \times 2 \mathrm{~mm}^{3}$ were cut from the metallic glass casts. The faces were prepared by grinding and polishing. The surfaces, in contact with the compression anvils, were carefully aligned to be parallel and were ground on a glass plate with diamond paste to avoid concavity of the surface.

The samples were preannealed at the testing temperature for an extended period. Samples for experiments at low stresses (Figs. 1, 2, and 7) were annealed for $5.2 \times 10^{5} \mathrm{~s}(550 \mathrm{~K}), \quad 2.3 \times 10^{5} \mathrm{~s}(555 \mathrm{~K})$, and 8.0 $\times 10^{4} \mathrm{~s}(565 \mathrm{~K})$, respectively. Samples for experiments at high stresses (Figs. 3, 5, and 6) were annealed for 1 $\times 10^{4} \mathrm{~s}(550$ and $555 \mathrm{~K})$, and $8.0 \times 10^{4} \mathrm{~s}(565 \mathrm{~K})$, respectively.

The plastic deformation tests were carried out in a Zwick Z050 uniaxial tester under compression. That tester had been modified by a feedback loop that controlled the load. Creep deformation was performed under constant true stress: the load $L$ was controlled to take into account the change of the cross section upon straining. For homogeneous plastic deformation at constant volume the true stress is 


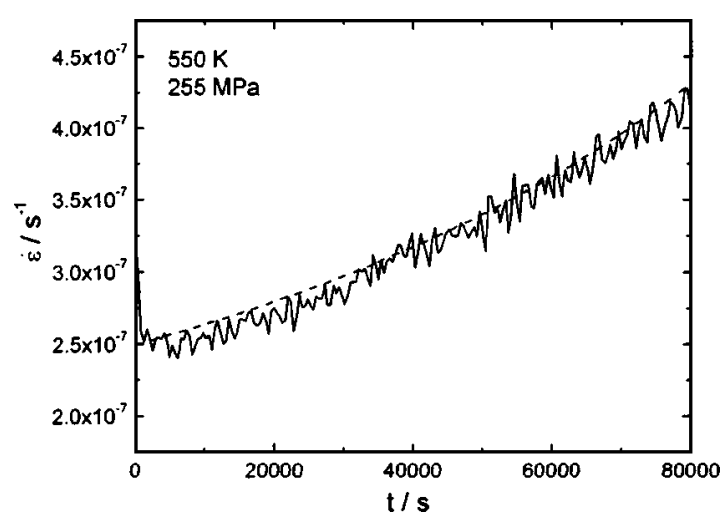

FIG. 2. Experimental (solid line) and calculated (dashed line) strain rates measured at $550 \mathrm{~K}$ and $255 \mathrm{MPa}$.

$$
\sigma_{\text {true }}=\frac{L}{A_{0}}(1-\varepsilon),
$$

where $A_{0}$ is the initial cross section and $\varepsilon$ is the nominal uniaxial strain. The duration of the individual creep tests was much greater than the decay time of the anelastic response that followed the load changes. The strain was measured directly at the sample by a linear inductive differential transducer at an accuracy of $\pm 10 \mathrm{~nm}$. Strain vs time data were taken at frequencies between 1 and $50 \mathrm{~Hz}$, depending on the duration of the experiments. The data were smoothed by averaging in bins of 100 points and differentiated. All data are presented as strain rate vs time.

\section{RESULTS}

Figure 1 shows the strain rate vs time in a sequence of tests at $550 \mathrm{~K}$, performed at successively higher true stresses of $30,60,90,119$, and $148 \mathrm{MPa}$. After the decay of the anelastic response following a stress change, the strain rate becomes constant, within the accuracy of the experiment. Such a constant strain rate is found in all tests below $255 \mathrm{MPa}$ at this temperature. Note that good data could be taken even at strain rates as low as $10^{-8} \mathrm{~s}^{-1}$.

Figures 2 and 3 show the results of subsequent tests at higher stresses: $255-636 \mathrm{MPa}$. An increasing strain rate is observed; at the highest stress, the increase is even stronger

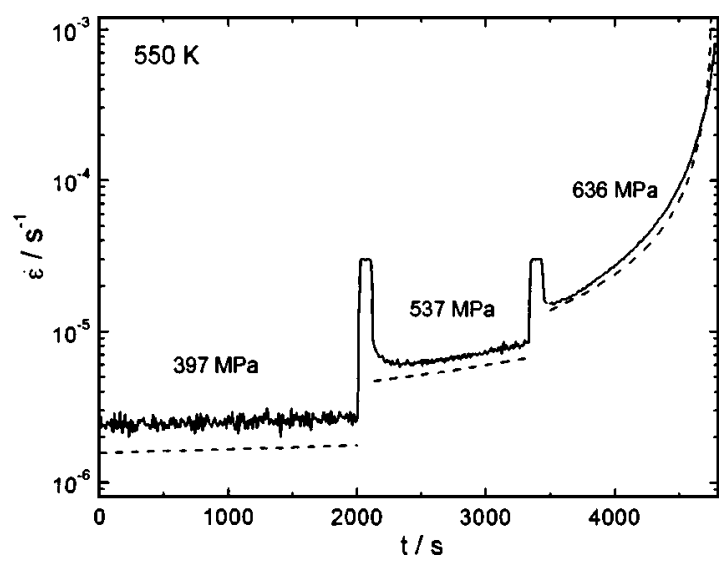

FIG. 3. Experimental (solid line) and calculated (dashed line) strain rates measured in a creep test at $550 \mathrm{~K}$ and 397, 537, and $636 \mathrm{MPa}$.
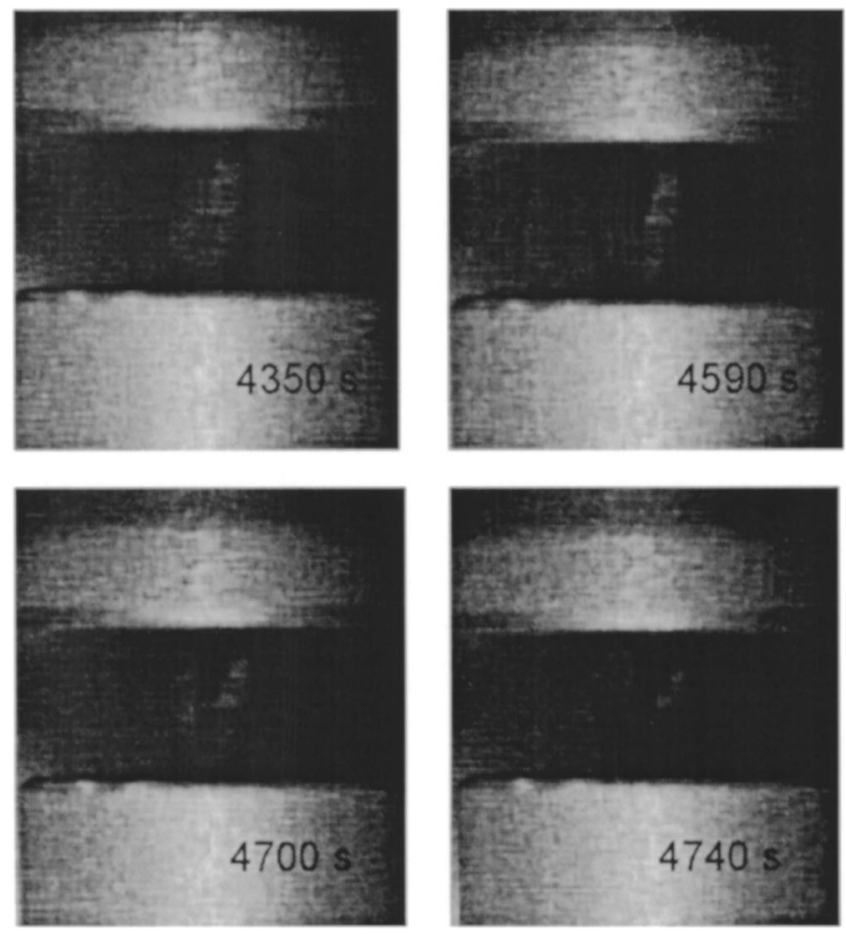

FIG. 4. Images taken from the video observation the sample during the test of Fig. 3. The times indicate that the images were taken from the test at the highest stress.

than exponential. Nevertheless, in all experiments, the deformation remains fully homogeneous, as is demonstrated by the sequence of images in Fig. 4, taken during the test at $636 \mathrm{MPa}$. The sample is oriented with one edge toward the front, so that two side faces can be seen. The upper and lower parts of the images are the compression anvils. During the final seconds of the test the sample appears to buckle a little bit, but the deformation remains homogeneous. No shear band can be seen. The sample fractured at high speed at the end of this test along a plane inclined $45^{\circ}$ to the compression axis. The fracture morphology showed the river pattern typical of the instability that develops in the lowviscosity material of a shear band. ${ }^{30}$ Failure occurred almost instantaneously after the formation of the shear band. The accelerating strain rate observed in the experiments is, therefore, a bulk property of the material: it is the result of stressinduced creation of flow defects. Figures 5 and 6 show that the same phenomenon is observed at other temperatures ( 555 and $565 \mathrm{~K}$ ).

Figure 6 also illustrates that the disordering process is reversible and reproducible. The solid line represents an accelerating strain rate during a test at $445 \mathrm{MPa}$ for $1700 \mathrm{~s}$. The sample was then tested for $5.8 \times 10^{4} \mathrm{~s}$ at $14 \mathrm{MPa}$ (not shown), during which the strain rate decreased because defect annihilation outstrips creation at low stresses. Subsequent testing at $445 \mathrm{MPa}$ (dashed line) again shows an accelerating strain rate (the steep initial drop is an anelastic transient irrelevant to this discussion). That the solid and dashed lines can be made to coincide by shifting their origin shows that the defect concentration in the third test again reaches its value in the first one, and subsequently evolves with the same kinetics. It is therefore justified to describe the 


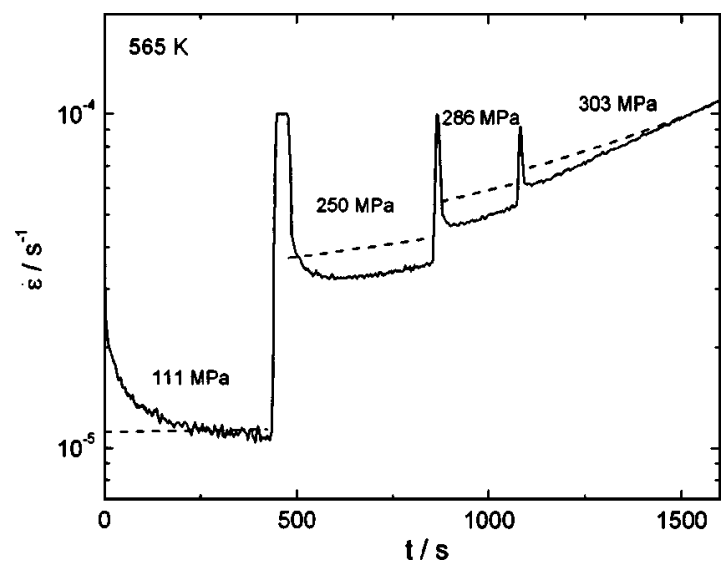

FIG. 5. Experimental (solid line) and calculated (dashed line) strain rates measured in a creep test at $565 \mathrm{~K}$ and $111,250,286$, and $303 \mathrm{MPa}$.

evolution of the defect concentration in terms of the instantaneous stress, strain rate, and defect concentration.

The black squares in Fig. 7 show the strain rates measured at the three temperatures for the lower stresses, at which a steady-state value is reached. The numbers indicate the order of the test. The arrows indicate the onset of acceleration of the strain rate. At low stress, the linear dependence of strain rate on stress, i.e., Newtonian viscous flow, is immediately apparent.

\section{ANALYSIS}

\section{A. Stress-dependence of the strain rate}

The solid lines in Fig. 7 are fits of Eq. (1) to the data. Table I lists the fitting parameters: the activation volume $\varepsilon_{0} \nu_{0}$ and the prefactor $2 k_{f} c_{f} \varepsilon_{0} \nu_{0} \Omega^{-1}$. The activation volume is independent of temperature.

Note that the data points at lower stress (e.g., 1-4 at $550 \mathrm{~K}$ ) lie generally slightly above the fit, whereas those in the middle range (e.g., 5-7 at $550 \mathrm{~K}$ ) lie slightly below it. This is caused by small changes in the defect concentration $c_{f}$ over the course of the experiments as a result of defect

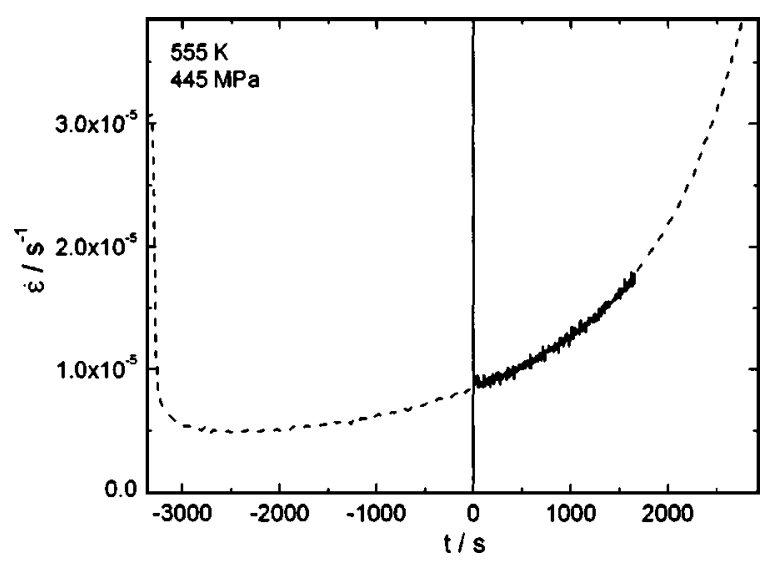

FIG. 6. Strain rates as a function of time measured at $555 \mathrm{~K}$. First experiment (solid line) at $445 \mathrm{MPa}$. Second experiment (not shown): $5.8 \times 10^{4} \mathrm{~s}$ at $14 \mathrm{MPa}$. Third experiment (dashed line) at $445 \mathrm{MPa}$. The origins of the two plotted lines were shifted to show the match.

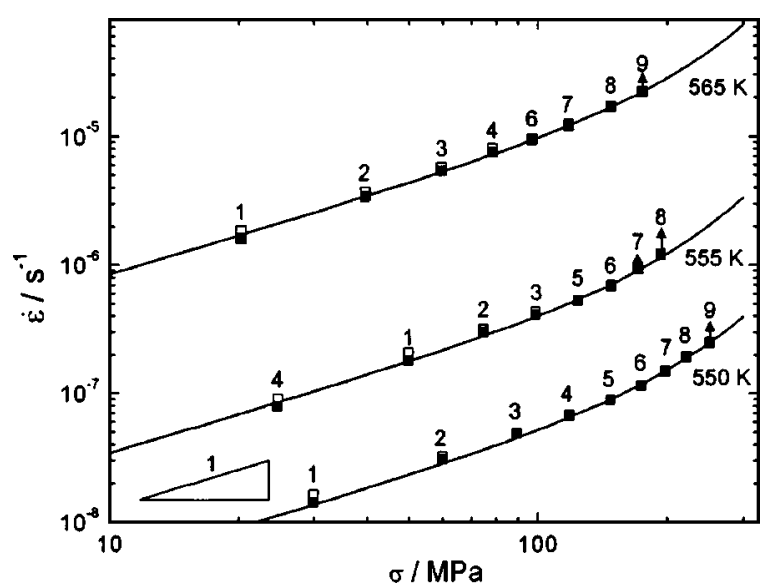

FIG. 7. Experimental (filled squares) and calculated (open squares) values of the stress dependence of the strain rate for low stresses at 550, 555, and $565 \mathrm{~K}$. Some of the open squares are obscured by perfect coincidence with the data. The arrows indicate that the strain rate increased from the initial value of the filled square. The triangle marks the slope for the linear relation. The solid line is the fit of all data at each temperature to Eq. (1). Test number 5 at $565 \mathrm{~K}$ (not shown) was made at $6 \mathrm{MPa}$.

annihilation and creation. The kinetics of these phenomena are analyzed in the following section, during which the analysis of Fig. 7 will be revisited.

\section{B. Kinetics of creation and annihilation}

\section{Strain-rate-dependent defect creation}

Based on the success of de Hey, Sietsma, and van den Beukel $^{6}$ with using strain-rate-dependent defect creation, as in Eq. (7), to model softening in tensile testing of $\mathrm{Pd}_{40} \mathrm{Ni}_{40} \mathrm{P}_{20}$ ribbons, we used this mechanism first to model our data. A combination of Eq. (2) and (7) gives the defect creation rate as

$$
\left.\frac{d c_{f}}{d t}\right|^{+}=a_{x} \dot{\varepsilon} c_{f}\left(\ln c_{f}\right)^{2} .
$$

For simplicity, we concentrated on the data at $550 \mathrm{~K}$, since the equilibrium defect concentration, according to de Hey, Sietsma, and van den Beukel ${ }^{6}$ is sufficiently low at this temperature that the simple bimolecular description, Eq. (3) can be used for defect annihilation.

The data were fit by numerically integrating Eq. (3) and (10) for the evolution of the defect concentration $c_{f}$ for each of the experimental conditions and by using the final value of $c_{f}$ in one test as the initial value for the next test. The following parameters were used: $\Omega=13.2 \AA^{3},{ }^{8} \varepsilon_{0} \nu_{0}=126 \AA^{3}$ [from a fit of Eq. (1) to just the first four data points at $550 \mathrm{~K}$ on Fig. 7], the initial value of $c_{f}$ was taken as $2 \times 10^{-14}$ (from de Hey, Sietsma, and van den Beukel $^{6}$ for the preannealing

TABLE I. Parameters used for the fits of Eq. (1) to the data in Fig. 7: the activation volume $\varepsilon_{0} \nu_{0}$ and the prefactor $2 k_{f} c_{f} \varepsilon_{0} \nu_{0} \Omega^{-1}$.

\begin{tabular}{clc}
\hline \hline$T(\mathrm{~K})$ & $\varepsilon_{0} \nu_{0}\left(\AA^{3}\right)$ & $2 k_{f} c_{f} \varepsilon_{0} \nu_{0} \Omega^{-1}\left(\mathrm{~s}^{-1}\right)$ \\
\hline 550 & $141.6 \pm 3.2$ & $4.9 \pm 0.2 \times 10^{-8}$ \\
555 & $152.0 \pm 10.8$ & $3.5 \pm 0.4 \times 10^{-7}$ \\
565 & $143.8 \pm 2.0$ & $4.6 \pm 0.1 \times 10^{-6}$ \\
\hline \hline
\end{tabular}




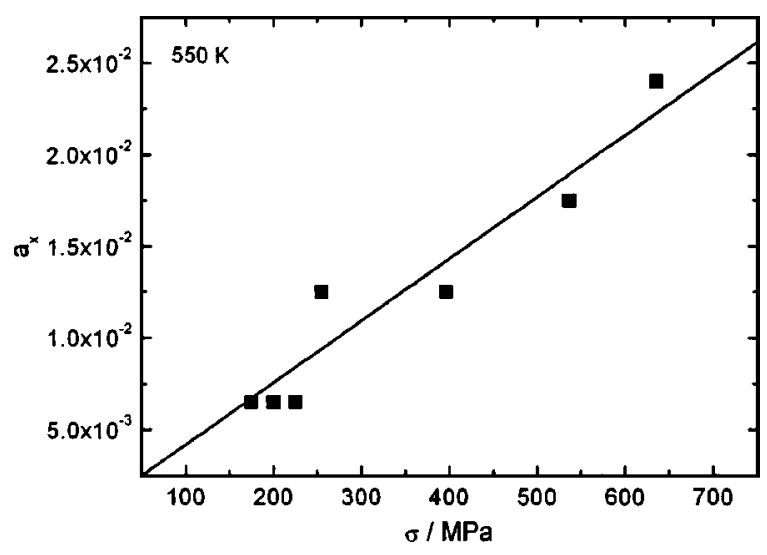

FIG. 8. Dependence of the proportionality constant for strain-rate-induced free volume production [Eq. (7)] on the stress.

conditions of our samples), and $k_{f}=2.3 \times 10^{5} \mathrm{~s}^{-1}$ [from the prefactor of the fit of Eq. (1) to the first four points]. The remaining parameters $k_{r}$ and $a_{x}$ were determined by fitting the data of Figs. 1-3. They could be varied only within narrow limits $(20 \%)$. A fit of the data with $k_{r}=9 \times 10^{7} \mathrm{~s}^{-1}$ gave parameters $a_{x}$ that depended strongly on the stress, as illustrated in Fig. 8. The dependence is clearly linear, which indicates that a defect creation mechanism that depends on the applied power density [Eq. (8)] is more appropriate here.

\section{Power-dependent defect creation}

The defect creation rate, derived from Eqs. (2) and (8), in this case is

$$
\left.\frac{d c_{f}}{d t}\right|^{+}=a_{x}^{\prime} \sigma \dot{\varepsilon} c_{f}\left(\ln c_{f}\right)^{2} .
$$

This equation was numerically integrated together with Eq. (4) for the defect annihilation rate. For fits at temperatures higher than $550 \mathrm{~K}$, it is necessary to take into account the approach of the defects to their equilibrium values $c_{\mathrm{eq}}$ which were taken from the determination by de Hey, Sietsma, and van den Beukel ${ }^{6}$ (see Table II). The initial defect concentration of the as cast material was estimated at $c_{\text {init }}=5 \times 10^{-14}$. In all cases, the defect evolution during preannealing was computed; this made the initial concentration for the creep tests insensitive to the precise estimate of the defect concentration in the as cast condition. As above, the final value of $c_{f}$

TABLE II. Parameters used to fit the creep data at 550, 555, and $565 \mathrm{~K}$; $\varepsilon_{0} \nu_{0}$, activation volume; $k_{f}$, jump frequency of the flow defects; $k_{r}$, rate constant for bimolecular annihilation of the defects; $a_{x}^{\prime}$, proportionality constant in the production of free volume; $c_{\text {init }}$, flow defect concentration of nonannealed samples; and $c_{\text {eq }}$, equilibrium density of flow defects.

\begin{tabular}{lccc}
\hline \hline & $550(\mathrm{~K})$ & $555(\mathrm{~K})$ & $565(\mathrm{~K})$ \\
\hline$\varepsilon_{0} \nu_{0}\left(\AA^{3}\right)$ & 106 & 106 & 106 \\
$k_{f}\left(\mathrm{~s}^{-1}\right)$ & $2.45 \times 10^{5}$ & $2.21 \times 10^{6}$ & $3.82 \times 10^{7}$ \\
$k_{r}\left(\mathrm{~s}^{-1}\right)$ & $5.15 \times 10^{7}$ & $2.67 \times 10^{8}$ & $3.31 \times 10^{9}$ \\
$a_{x}^{\prime}\left(\mathrm{Pa}^{-1}\right)$ & $5.05 \times 10^{-11}$ & $3.61 \times 10^{-11}$ & $1.88 \times 10^{-11}$ \\
$c_{\text {init }}$ & $5 \times 10^{-14}$ & $5 \times 10^{-14}$ & $5 \times 10^{-14}$ \\
$c_{\text {eq }}$ & $2 \times 10^{-15}$ & $8 \times 10^{-15}$ & $2.1 \times 10^{-14}$ \\
$($ de Hey et al. 1998$)$ & & & \\
\hline \hline
\end{tabular}

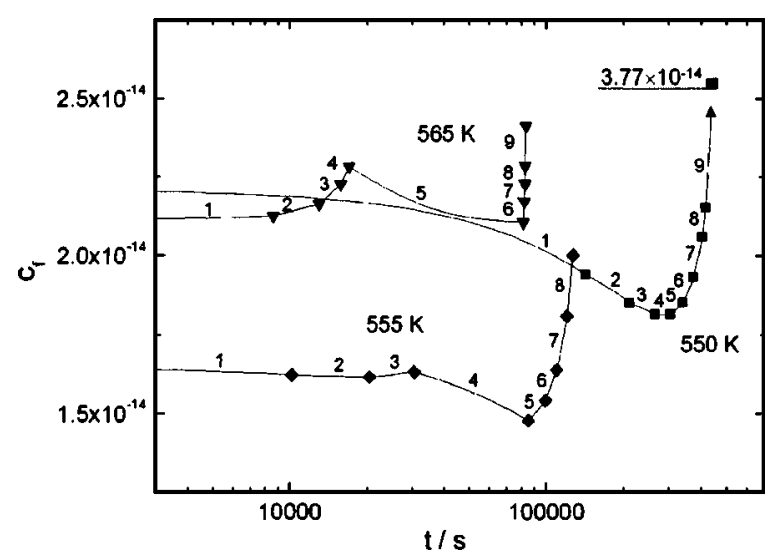

FIG. 9. Calculated evolution of the defect concentration over the course of three test sequences, at 550 (squares), 555 (diamonds), and $565 \mathrm{~K}$ (triangles).

for each test was taken as the input value for the next test. The fit parameters for all the tests are listed in Table II. A single value of the activation volume $\varepsilon_{0} \nu_{0}$ was used; it was found that $\varepsilon_{0} \nu_{0}=106 \AA^{3}$ gave the best results. That this value is considerably smaller than that found in the fits of Sec. I reflects the effect of the small changes in defect concentration in those data.

The open squares in Fig. 7 are the values calculated by the model for the tests at low stress; the agreement is generally very good (the calculated and experimental values are often indistinguishable). The calculation also reproduces the slight deviations of the data points from the overall fit (e.g., the first four points at $550 \mathrm{~K}$ being above the line). This shows that these deviations are indeed the result of small changes in defect concentration between the tests.

Figure 9 shows the calculated evolution of the defect concentration for the tests at low stress. The numbers of the individual tests correspond to those in Fig. 7. The points in Fig. 9 correspond to the beginning of each test. At $550 \mathrm{~K}$, in test 4 at $119 \mathrm{MPa}$, the defect concentration $c_{f}$ remains nearly constant; at lower stresses, it decreases; at higher stresses it increases. This is analyzed further in Fig. 10, which shows two plots (linear and $\log -\log$ ) of $d c_{f} / d t$ as a function of $c_{f}$ at 90, 119, and $148 \mathrm{MPa}$, the stresses of tests 3, 4, and 5. At $119 \mathrm{MPa}$, the curve crosses zero at $c_{f}=1.8 \times 10^{-14}$, which is the steady-state value seen in Fig. 9. At $90 \mathrm{MPa}$, the steadystate value is $4.3 \times 10^{-15}$; as a result, if creep test 3 were continued, the strain rate would decrease over a very long time by about a factor of 5 . At $148 \mathrm{MPa}$, the steady-state value is $4.6 \times 10^{-12}$ and under the conditions of the test the defect creation rate even accelerates. The steady-state values of $c_{f}$ for all stresses are shown in Fig. 11; the steady-state value at zero stress is, of course, the equilibrium concentration. Figure 12 is a calculated creep test at $148 \mathrm{MPa}$. It shows that steady state is reached only after $3.1 \times 10^{6} \mathrm{~s}$ and $1000 \%$ strain, which is unrealistic. Under ordinary laboratory conditions only a continuously accelerating strain rate is seen in experiments at high stress.

In the tests at 555 and $565 \mathrm{~K}$, the tests were not performed in an ascending series of stresses; test 4 at $555 \mathrm{~K}$ and test 5 at $565 \mathrm{~K}$ were at lower stresses. During these tests the 


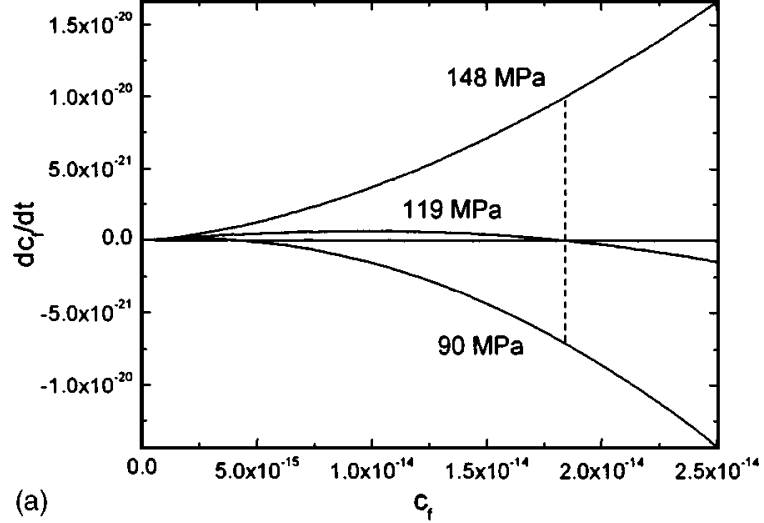

(a)

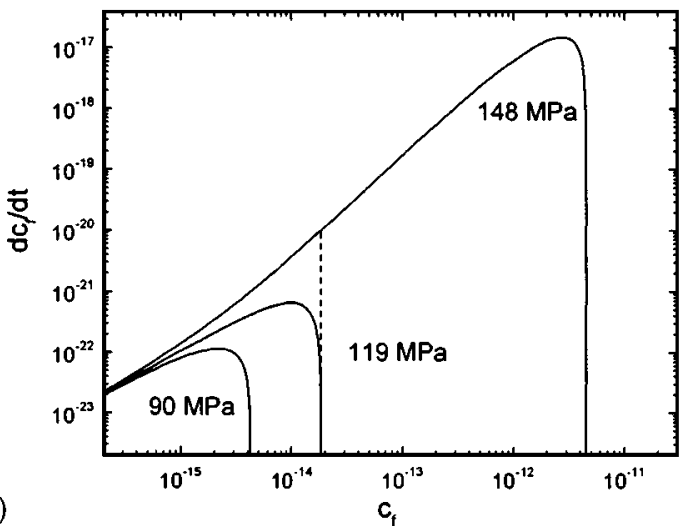

FIG. 10. Linear (a) and logarithmic (b) plots of the rate of change of the defect concentration as a function of the defect concentration, calculated using Eqs. (4) and (11) for the conditions of the test sequence at $550 \mathrm{~K}$ on Fig. 9; the vertical dashed line corresponds to the defect concentration in test 4.

defect concentration drops. As a result the strain rates (experimental and calculated) in subsequent tests tend to lie below the overall fit.

The test of Fig. 2 (550 K,255 MPa), which exhibits an accelerating strain rate, is reproduced well by the model (dashed line). In other tests at high stress (Figs. 3 and 5), the agreement is less perfect. The strongly accelerating strain rate at the highest stress (636 MPa, Fig. 3) is reproduced. The discrepancies between fits and data may be the result of the transients corresponding to the stress changes. During

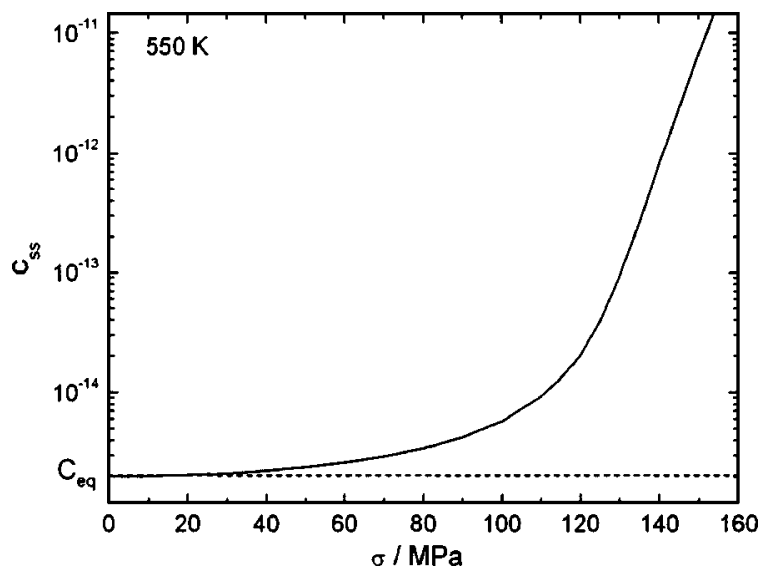

FIG. 11. The steady state defect concentration as a function of stress, calculated for the conditions of Fig. 10.

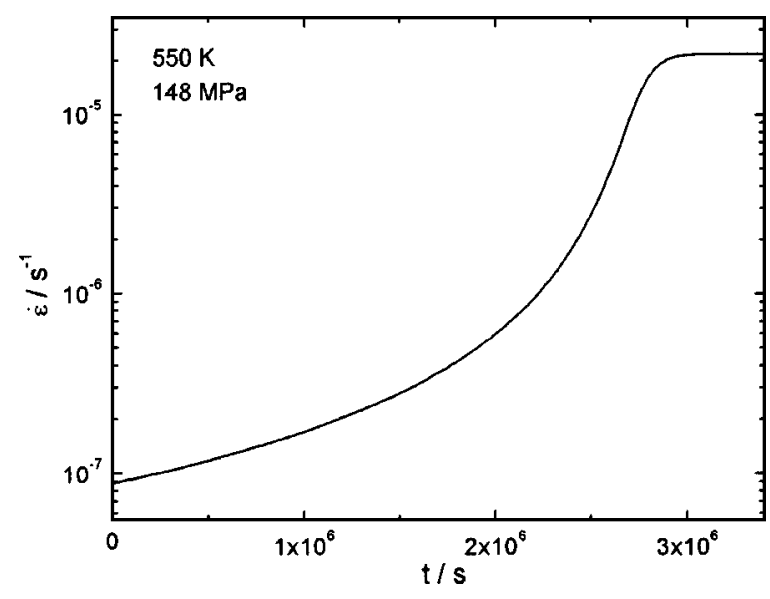

FIG. 12. Strain rate as a function of time calculated for a long-term test under the conditions of test 5 in Figs. 9 and $10(550 \mathrm{~K}, 148 \mathrm{MPa})$.

these transients, which can take substantial amounts of time, the defect concentration may change considerably, but cannot be calculated.

\section{DISCUSSION}

The quantitative demonstration in this paper of pronounced deformation-induced defect generation during homogeneous flow has several interesting implications. For the experimentalists, it brings out the importance of knowing the true stress in a test, especially at high stresses, where the strain rate can depend strongly on the true stress. Creep experiments at constant nominal stress, ${ }^{31}$ for example, can give erroneous results in this regime.

Our observations and measurements also unambiguously show that the accelerating strain rate is an intrinsic feature of homogeneous flow and not an artifact of the geometry. In tensile creep experiments, ${ }^{25,32}$ rapid increases in the strain rate prior to failure have been attributed to void formation or necking, both of which are ruled out in our compression geometry. In our experiments, shear band formation and failure occurred during a small fraction of the very last second.

The activation volume for flow found in these experiments is $\varepsilon_{0} \nu_{0}=106 \AA^{3}$, which is of the same magnitude but smaller that the value $\varepsilon_{0} \nu_{0}=130 \AA^{3}$ measured in the work of de Hey, Sietsma, and van den Beukel ${ }^{6}$ in tensile testing of $\mathrm{Pd}_{40} \mathrm{Ni}_{40} \mathrm{P}_{20}$ ribbons. Since the average atomic volume in our alloy is $\Omega=13.2 \AA^{3}$, the activation volume found corresponds to eight atomic volumes. Since these experiments only give the product $\varepsilon_{0} \nu_{0}$, the size of the flow defect can not be determined accurately. It seems plausible, however, that it consists of at least eight atoms, since local strains greater than unity have not been seen in any model simulations.

Figure 13(a) is an Arrhenius plot of the rate constant for flow rearrangements $k_{f}$. The corresponding activation energy is $9.03 \pm 0.64 \mathrm{eV}$. As Eq. (1) shows, this corresponds to the activation energy for isoconfigurational flow, i.e., flow at constant structure and hence constant defect concentration $c_{f}$. Under these conditions $k_{f}$ is the dominant temperaturedependent factor in Eq. (1). Isoconfigurational flow has been achieved in creep experiments far below the glass transition temperature by rapidly cycling the testing temperature. In 


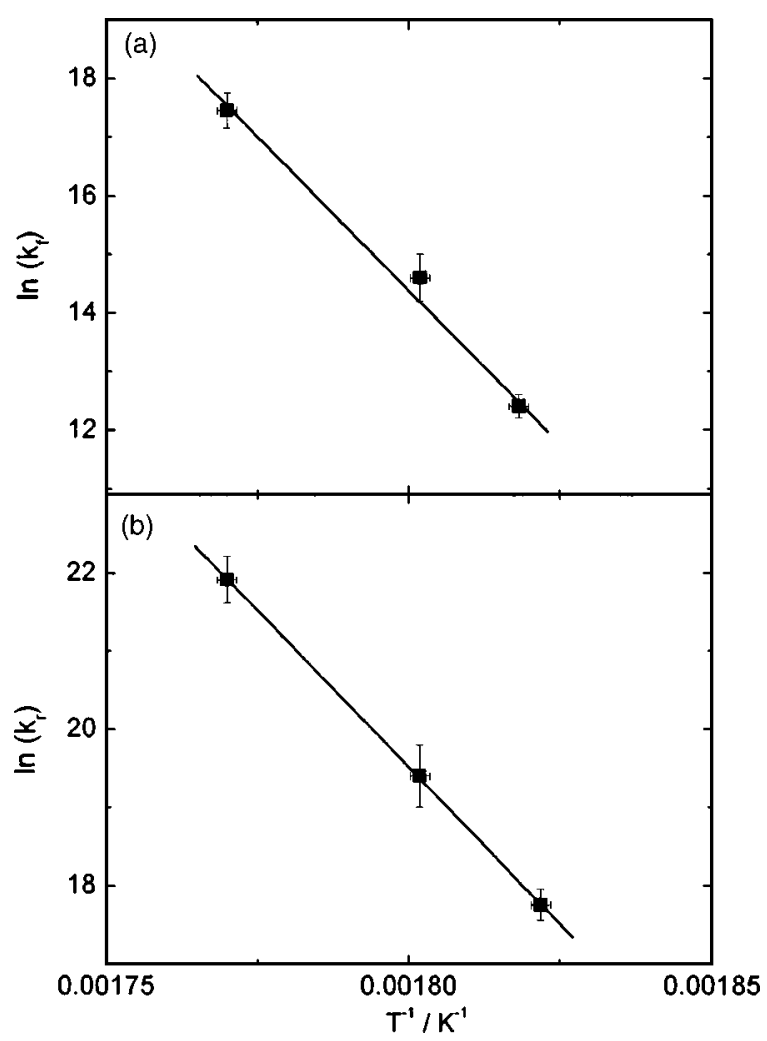

FIG. 13. (a) Arrhenius plot of the temperature dependence of $k_{f}$, the rate constant for flow [Eq. (1)]; activation energy $9.03 \pm 0.64 \mathrm{eV}$. (b) Arrhenius plot of the temperature dependence of $k_{r}$, the rate constant for defect annihilation [Eq. (4)]; activation energy 7.44 $\pm 0.64 \mathrm{eV}$.

$\mathrm{Pd}_{82} \mathrm{Si}_{18}$, the activation energy for isoconfigurational flow was found to be $2.0 \mathrm{eV}^{25}$ The large discrepancy between these values has two implications. First, during the temperature-cycling experiments some reversible structural changes must have occurred (e.g., as a result of thermal expansion) that altered the defect concentration. Subsequent work on isoconfiguration flow in $\mathrm{Pd}_{77.5} \mathrm{Cu}_{6} \mathrm{Si}_{16.5}$ already indicated that such changes could be occurring. ${ }^{19}$ Second, the value of the activation energy found here is too high to be attributable to a singly activated event: it far exceeds even the cohesive energy. The shear of a flow defect, therefore, consists probably of a sequence of several activated events, which is plausible given that the defect consists of several atoms.

Figure 13(b) is an Arrhenius plot of the rate constant for bimolecular defect annihilation $k_{r}$. The corresponding activation energy is $7.44 \pm 0.64 \mathrm{eV}$. That this value is close to the activation energy for $k_{f}$ is consistent with earlier observations that the activation energy for the rate of change of the viscosity $\dot{\eta}$ measured in earlier tensile creep work is quite small, ${ }^{21,25}$ on the order of $-0.1 \mathrm{eV}$. A comparison of Eqs. (1) and (3) shows that $\dot{\eta} \propto k_{r} / k_{f}$, so that its activation energy should be the difference between that of the two rate constants. In these experiments, that difference has the right sign, and, using the outer values of the (standard deviation) error bars, a plausible magnitude.

Figure 14 is a plot of the temperature dependence of $a_{x}^{\prime}$. Its numerical values can be checked against the simple model described in the introduction, which leads to the definition of $a_{x}^{\prime}$ from Eq. (8),

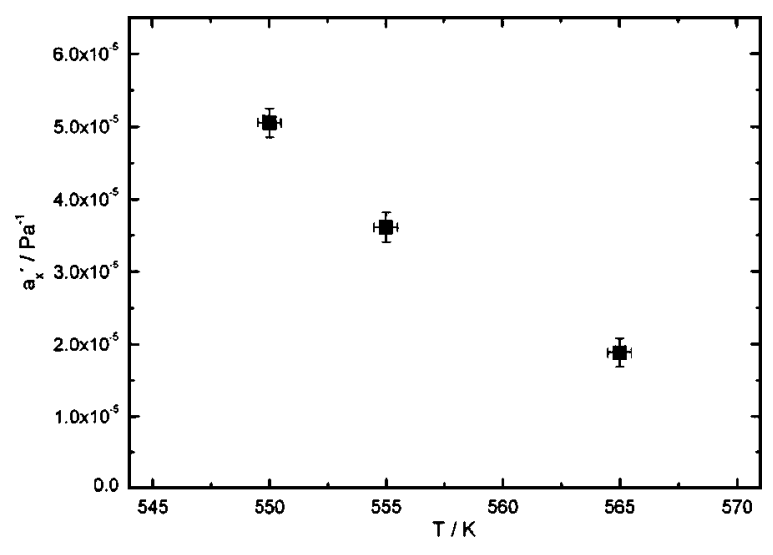

FIG. 14. Temperature dependence of the proportionality constant in the power-density-induced production of free volume [Eq. (8)].

$$
a_{x}^{\prime}=\frac{\Omega}{8 S \nu_{f}} .
$$

Equation (2) gives the relation between $c_{f}$ and $v_{f}$. With the value $\gamma \nu^{*}=0.143 \Omega$, as determined by van den Beukel and Sietsma, ${ }^{22}$ and the observation that most of the defect concentrations are on the order of $c_{f} \sim 2 \times 10^{-14}$ (see Fig. 9), we estimate $v_{f} \sim 0.0045 \Omega$. Since the modulus $S$ for Pd-based alloys is $51 \mathrm{GPa},{ }^{4}$ Eq. (12) gives $a_{x}^{\prime}=5.4 \times 10^{-10} \mathrm{~Pa}^{-1}$. That the experimental values are an order of magnitude smaller means that only a small fraction of the volume produced in the dilatation mechanism is retained as free volume.

The energy cost of free volume production has been estimated by van den Beukel and Sietsma from calorimetric studies of structural relaxation. ${ }^{22}$ The production of free volume requires energy according to $(\Delta U)_{f v}=\beta \Delta x$. For amorphous $\mathrm{Pd}_{77.5} \mathrm{Cu}_{6} \mathrm{Si}_{16.5}$ a value $\beta=0.41 \mathrm{eV}$ was estimated. The quantity $\beta^{\prime}=\beta \Omega / \gamma \nu^{*}$ is the formation energy of one atomic volume's worth of free volume. ${ }^{22}$ For $\gamma \nu^{*}=0.143 \Omega, \beta^{\prime}$ $=2.90 \mathrm{eV}$. Using the parameter $a_{x}^{\prime}$ from our fits, we can calculate a proportionality factor $\beta_{W}$ between the mechanical power applied on the sample and the reduced free volume according to $\Delta W=\beta_{W} \Delta x$, with $\beta_{W}^{\prime}=\beta_{W} \Omega / \gamma \nu^{*}$. The results for 550,555 , and $565 \mathrm{~K}$ are compiled in Table III. The factor $\beta_{W}^{\prime}$ represents the mechanical work necessary to form one atomic volume's worth of free volume. The resulting values of $\beta_{W}^{\prime}$ are distinctly higher than the thermal formation energy $\beta^{\prime}$. The ratio $\beta_{W}^{\prime} / \beta^{\prime}$ is the mechanical efficiency of the formation process $9 \%-24 \%$ of the applied energy is used to form the vacancy, the rest is dissipated as heat. This inefficiency is hardly surprising: the same applies to the creation of dislocations in the deformation of crystals.

It should be kept in mind, of course, that the disordering mechanism described in the introduction needs to be adjusted in view the current results. The temperature depen-

TABLE III. Proportionality factor between mechanical energy and reduced free volume $\beta_{W}$ and free volume $\beta_{W^{\prime}}$, respectively, for 550,555 , and $565 \mathrm{~K}$.

\begin{tabular}{lccc}
\hline \hline$(\mathrm{eV})$ & $550(\mathrm{~K})$ & $555(\mathrm{~K})$ & $565(\mathrm{~K})$ \\
\hline$\beta_{W}$ & 1.72 & 2.37 & 4.56 \\
$\beta_{W}^{\prime}$ & 12.0 & 16.6 & 31.9 \\
\hline \hline
\end{tabular}


dence of $a_{x}^{\prime}$ (Fig. 14), for example, is stronger than predicted by Eq. (12). Needed is a kinematic model for dilatation of a flow defect that goes beyond the motion of a single atom and takes into account the cooperative motion of the all the atoms in the flow defect.

\section{CONCLUSION}

We have obtained quantitative evidence for strong deformation-induced disordering of a metallic glass during homogeneous flow. Because of the possibly strong dependence of the strain rate on the stress, precise knowledge of the true stress in the creep tests is essential. Measurements of the activation volume and of the activation energy for isoconfigurational flow indicate that a flow defect consists of several atoms. The disordering rate was shown to be proportional to the applied power density, as postulated by Falk and Langer. ${ }^{5}$ Interpretation of the proportionality factor according to an earlier free volume model showed the disordering process to be "inefficient," in that only a fraction of the dilated volume is preserved as free volume and most of the applied power is dissipated as heat.

\section{ACKNOWLEDGMENTS}

The authors are grateful to Dr. Jan Schroers (Caltech) and Professor Dieter Herlach (DLR, Köln) for providing the samples. F.S. acknowledges support by a Senior Research Award from the Alexander von Humboldt-Stiftung and is grateful to Professor Knut Urban for his hospitality; the author's work in this area at Harvard is supported by the MRSEC program of the National Science Foundation.

${ }^{1}$ F. Spaepen, in Defects in Amorphous Metals, Les Houches Lectures XXXV on Physics of Defects edited by R. Balian et al. (North-Holland,
Amsterdam, 1981), p. 133

${ }^{2}$ F. Spaepen and A. I. Taub, Flow and Fracture, in Amorphous Metallic Alloys, edited by F. E. Luborsky (Butterworths, London, 1983), pp. 231.

${ }^{3}$ P. S. Steif, F. Spaepen, and J. W. Hutchinson, Acta Metall. 30, 447 (1982). ${ }^{4}$ F. Spaepen, Acta Metall. 25, 407 (1977).

${ }^{5}$ M. L. Falk and J. S. Langer, Phys. Rev. E 57, 7192 (1998).

${ }^{6}$ P. de Hey, J. Sietsma, and A. van den Beukel, Acta Mater. 46, 5873 (1998).

${ }^{7}$ W. L. Johnson, J. Lu, and M. D. Demetriou, Intermetallics 10, 1039 (2002).

${ }^{8}$ J. Lu, G. Ravichandran, and W. L. Johnson, Acta Mater. 51, 3429 (2003). ${ }^{9}$ M. Heggen, F. Spaepen, and M. Feuerbacher, Mater. Sci. Eng., A 375377, 366 (2004).

${ }^{10}$ M. Heggen, F. Spaepen, and M. Feuerbacher, Mater. Res. Soc. Symp. Proc. 805, MM7.2.1 (2004).

${ }^{11}$ M. Bletry, P. Guyot, Y. Bréchet, J. J. Blandin, and J. L. Soubeyroux, Mater. Sci. Eng., A (in press).

${ }^{12}$ M. D. Demetriou and W. L. Johnson, J. Appl. Phys. 95, 2857 (2004).

${ }^{13}$ R. W. Cahn, N. A. Pratten, M. G. Scott, H. R. Sinning, and L. Leonardson, Symposium on Rapidly Solidified Materials, edited by B. H. Kear and B.

C. Giessen (North-Holland, Amsterdam, 1984), p. 241.

${ }^{14}$ H. S. Chen and S. Y. Chuang, Appl. Phys. Lett. 27, 316 (1975).

${ }^{15}$ P. E. Donovan and W. M. Stobbs, Acta Metall. 29, 1419 (1981).

${ }^{16}$ J. Li, F. Spaepen, and T. C. Hufnagel, Philos. Mag. A 82, 2623 (2002).

${ }^{17}$ O. Reynolds, Philos. Mag. 20, 469 (1885).

${ }^{18}$ D. W. Taylor, Fundamentals of Soil Mechanics (Wiley, New York, 1948).

${ }^{19}$ S. S. Tsao and F. Spaepen, Acta Metall. 33, 881 (1985).

${ }^{20}$ C. A. Volkert and F. Spaepen, Mater. Sci. Eng. 97, 449 (1988).

${ }^{21}$ C. A. Volkert and F. Spaepen, Acta Metall. 37, 1355 (1989).

${ }^{22}$ A. van den Beukel and J. Sietsma, Acta Metall. Mater. 38, 383 (1990).

${ }^{23}$ A. van den Beukel, Acta Metall. Mater. 39, 2709 (1991).

${ }^{24}$ M. H. Cohen and D. J. Turnbull, J. Chem. Phys. 31, 1164 (1959).

${ }^{25}$ A. I. Taub and F. Spaepen, Acta Metall. 28, 1781 (1980).

${ }^{26}$ A. Witvrouw and F. Spaepen, J. Appl. Phys. 74, 7154 (1993).

${ }^{27}$ F. Spaepen, Mater. Sci. Eng., A 179/180, 81 (1994).

${ }^{28}$ P. A. Duine, J. Sietsma, and A. van den Beukel, Acta Metall. Mater. 40, 743 (1992).

${ }^{29}$ J. D. Eshelby, Philos. Trans. R. Soc. London A244, 87 (1951).

${ }^{30}$ F. Spaepen, Acta Metall. 23, 615 (1975).

${ }^{31}$ H. Kato, A. Inoue, and H. S. Chen, Appl. Phys. Lett. 79, 4515 (2001).

${ }^{32}$ J. C. Gibeling and W. D. Nix, Scr. Metall. 12, 919 (1978). 